\title{
OPEN Agreement between dried blood spots and HemoCue in Tamil Nadu, India
}

\author{
Reshma P. Roshania ${ }^{1}$, Rukshan V. Mehta ${ }^{1}$, Ashwini Shete ${ }^{2}$, Rohini Bingewar ${ }^{2}$, \\ Sangeeta Kulkarni ${ }^{2}$, Aprajit Mahajan ${ }^{3}$, Grant Miller ${ }^{4}$, Alessandro Tarozzi ${ }^{5}$ \& \\ Reynaldo Martorell ${ }^{1,6 \bowtie}$
}

India retains the world's largest burden of anemia despite decades of economic growth and anemia prevention programming. Accurate screening and estimates of anemia prevalence are critical for successful anemia control. Evidence is mixed on the performance of HemoCue, a point-of-care testing device most widely used for large-scale surveys. The use of dried blood spots (DBS) to assess hemoglobin $(\mathrm{Hb})$ concentration is a potential alternative, particularly in field settings. The objective of this study is to assess $\mathrm{Hb}$ measurement agreement between capillary HemoCue and DBS among two age groups, children 6-59 months and females age 12-40 years. We analyzed data from the baseline round of a cluster randomized rice fortification intervention in Cuddalore district of Tamil Nadu, India. Capillary blood was collected from a subset of participants for $\mathrm{Hb}$ assessment by HemoCue 301 and DBS methods. We calculated Lin's concordance correlation coefficient, and tested bias by conducting paired t-tests of $\mathrm{Hb}$ concentration. Independence of the bias and $\mathrm{Hb}$ magnitude was examined visually using Bland-Altman plots and statistically tested by Pearson's correlation. We assessed differences in anemia classification using McNemar's test of marginal homogeneity. Concordance between HemoCue and DBS $\mathrm{Hb}$ measures was moderate for both children 6-59 months $\left(\rho_{c}=0.67 ; 95 \% \mathrm{Cl} 0.65\right.$, $0.71)$ and females $12-40$ years $\left(\rho_{c}=0.67: 95 \% \mathrm{Cl} 0.64,0.69\right)$. HemoCue measures were on average $0.06 \mathrm{~g} / \mathrm{dL}$ higher than DBS for children $(95 \% \mathrm{Cl} 0.002,0.12 ; \mathrm{p}=0.043)$ and $0.29 \mathrm{~g} / \mathrm{dL}$ lower than DBS for females $(95 \% \mathrm{Cl}-0.34,-0.23 ; \mathrm{p}<0.0001) .50 \%$ and $56 \%$ of children were classified as anemic according to HemoCue and DBS, respectively ( $p<0.0001) .55 \%$ and $47 \%$ of females were classified as anemic according to HemoCue and DBS, respectively $(p<0.0001)$. There is moderate statistical agreement of $\mathrm{Hb}$ concentration between HemoCue and DBS for both age groups. The choice of $\mathrm{Hb}$ assessment method has important implications for individual anemia diagnosis and population prevalence estimates. Further research is required to understand factors that influence the accuracy and reliability of DBS as a methodology for $\mathrm{Hb}$ assessment.

\author{
Abbreviations \\ BFA Body mass index-for-age z-score \\ BMI Body mass index \\ CCC Concordance correlation coefficient \\ CI Confidence interval \\ CMG Cyanmethemoglobin \\ DBS Dried blood spots \\ FPS Fair price shop \\ g/dL Grams per deciliter \\ HAZ Height-for-age z-score \\ $\mathrm{Hb} \quad$ Hemoglobin
}

\footnotetext{
${ }^{1}$ Nutrition and Health Sciences, Laney Graduate School, Emory University, Atlanta, GA, USA. ${ }^{2}$ National AIDS Research Institute, Indian Council of Medical Research, Pune, Maharashtra, India. ${ }^{3}$ Department of Agricultural and Resource Economics, University of California Berkeley, Berkeley, CA, USA. ${ }^{4}$ Center for Health Policy/Center for Primary Care and Outcomes Research, School of Medicine, Stanford, CA, USA. ${ }^{5}$ Department of Economics and Business, Universitat Pompeu Fabra and Barcelona GSE, Barcelona, Spain. ${ }^{6}$ Hubert Department of Global Health, Rollins School of Public Health, Emory University, 1518 Clifton Road, NE, CNR 5005, Mailstop \#1518-002-7BB, Atlanta, GA 30322, USA. ${ }^{\square}$ email: rmart77@emory.edu
} 
ICMR Indian Council of Medical Research

IFMR Institute of Financial Management and Research

JPAL Abdul Latif Jameel Poverty Action Lab

LoA Limits of agreement

NARI National AIDS Research Institute

SC/ST Scheduled caste/scheduled tribe

SD Standard deviation

WHZ Weight-for-height z-score

The consequences of anemia, defined as low hemoglobin $(\mathrm{Hb})$ concentration, are significant and many, including poor maternal and perinatal outcomes such as maternal mortality, low birth weight and pre-term birth; longer term child health outcomes such as impaired cognitive development; and broader socioeconomic costs due to reduced work potential ${ }^{1}$. India has a severe burden of anemia; the prevalence of anemia among children under five is approximately $40 \%^{2}$, and over one in two women of reproductive age (15-49 years) are anemic ${ }^{3}$.

Despite longstanding anemia prevention programming and recent decades of economic growth, there has been little progress in reducing the prevalence of anemia in India ${ }^{4}$, with trends in some states showing increases in anemia burden ${ }^{5}$. A key component of successful anemia control is accurate diagnosis. The gold standard for the assessment of $\mathrm{Hb}$ concentration is analysis of venous blood using the direct cyanmethemoglobin (CMG) method $^{6}$. This method, however, is not always feasible for survey research as venous blood collection is invasive, must be conducted by skilled personnel, and spectrophotometric analysis in a laboratory is required within hours of specimen collection.

HemoCue, a portable, point-of-care testing device that reads $\mathrm{Hb}$ in whole capillary, venous or arterial blood by spectrophotometry is widely used for anemia prevalence estimation in population-based surveys. In field settings, collection of capillary blood is most feasible, although biological variability between capillary and venous $\mathrm{Hb}$ concentration is likely ${ }^{7}$. Existing evidence demonstrates that accuracy of HemoCue capillary blood samples is generally poorer compared to the accuracy of venous and arterial HemoCue samples ${ }^{8}$. One review of HemoCue accuracy among children concluded that research is lacking in field-based and primary care settings in low resource contexts ${ }^{9}$. Furthermore, HemoCue performance can vary by specific model based on preanalytical factors related to the context such as temperature and humidity, as well as factors related to measurement protocol such as time from specimen collection to reading ${ }^{10}$. Adherence to quality control procedures during data collection such as using microcuvettes from newly opened containers and ensuring complete microcuvette filling are also critical for accurate HemoCue readings ${ }^{11}$.

An alternate field-friendly method of $\mathrm{Hb}$ collection and assessment is indirect CMG using dried blood spots (DBS), for which capillary blood is collected on filter paper, dried, and transported to a laboratory where samples are extracted with a solvent before analysis. There are several advantages to this approach compared to the gold standard ${ }^{12}$. Similar to HemoCue, sampling is less invasive and equipment is portable; additionally, DBS is less costly than HemoCue and samples can remain at room temperature for several days before analysis. Unlike HemoCue however, $\mathrm{Hb}$ measures from DBS are not immediately available and must be analyzed in a laboratory. We found only one study comparing indirect CMG using DBS of capillary blood to the gold standard of direct $\mathrm{CMG}$ of venous blood, which reported that the indirect method overestimates anemia prevalence. $\mathrm{Hb}$ measures using DBS were on average $0.7 \mathrm{~g} / \mathrm{dL}$ lower than direct $\mathrm{CMG}^{13}$. This study also compared indirect CMG using DBS to HemoCue and found that DBS measures were on average, $0.9 \mathrm{~g} / \mathrm{dL}$ lower than for HemoCue, increasing anemia prevalence by 24 percentage points, from $14 \%$ by HemoCue to $38 \%$ by DBS. The authors suggest incomplete dissolution of blood from filter paper as a potential explanation of these findings. This conclusion has been challenged by Mohanram and colleagues however, who report a similar pattern despite ensuring complete dissolution of blood from DBS samples; capillary Hb values measured using DBS were approximately $2.0 \mathrm{~g} / \mathrm{dL}$ lower than HemoCue for both pregnant and non-pregnant women in a hospital setting ${ }^{14}$. Lower DBS readings compared to HemoCue have also been reported for venous blood comparisons; a hospital-based study among adults in rural India found that $\mathrm{Hb}$ concentration values from indirect CMG using DBS of venous blood are $1.8 \mathrm{~g} / \mathrm{dL}$ lower compared to venous HemoCue $\mathrm{e}^{15}$.

The comparisons between HemoCue and indirect CMG using DBS have only been examined in adult populations. Differences in $\mathrm{Hb}$ measurement concordance by age group have been observed when comparing HemoCue to a reference method ${ }^{16}$, suggesting that age may be an important consideration in the choice and assessment of $\mathrm{Hb}$ measurement methods. The objective of this study is to evaluate the extent of agreement between two methods of $\mathrm{Hb}$ estimation that are feasible in field settings, capillary HemoCue and indirect CMG using DBS, among different age groups that are critical to consider to prevent the long-term and intergenerational consequences of anemia-young children, and adolescent and adult non-pregnant females.

\section{Methods}

This cross-sectional study analyzed the baseline round of data from a cluster randomized rice fortification intervention in Tamil Nadu, India. The research project (clinicaltrials.gov, NCT 03573570) is jointly funded by the Government of Tamil Nadu, Global Innovation Fund, the Tata Trusts and King Philanthropies; led by investigators at Stanford University, University of California Berkeley, Universitat Pompeu Fabra, and Emory University; and carried out in collaboration with the Abdul Latif Jameel Poverty Action Lab (JPAL) South Asia at the Institute of Financial Management and Research (IFMR). All methods were performed in accordance with the approved protocol and relevant regulations. 
Study population. Baseline data were collected between July and October 2018 in the catchment areas of 223 randomly selected Fair Price Shops (FPS) across predominantly rural Chidambaram and Bhuvanagiri taluks (administrative subdivisions) in Cuddalore District. The Government of India Public Distribution System operates through FPS by providing a monthly quota of subsidized staples; in Tamil Nadu this includes rice, wheat and sugar to households with ration cards. Selected FPS were randomized to receive either nutrient fortified or regular rice. Within each FPS area, households with active FPS ration cards were sampled randomly until a target of 40 non-pregnant females 12-40 years and 40 children 6-59 months were reached. In the overall study, a total of 7320 eligible households were surveyed, containing 6646 children 6-59 months and 8076 females 12-40 years. Both HemoCue and DBS samples were available for a random subset of all study participants (ten females 12-40 years and ten children 6-59 months per FPS area); this subset was included in our analysis, further described below.

Review of literature. We searched PubMed and Google Scholar for published studies on the validity of HemoCue and DBS methods as compared to the gold standard of direct CMG, and studies comparing HemoCue with DBS of both capillary and venous blood. We reviewed studies in resource-limited contexts, from both community and hospital settings.

Data collection and Hb assessment. HemoCue and DBS were collected at a fixed location, a health camp set up by the survey team in each FPS area. After completion of consenting protocols and a household survey questionnaire, participants visited the health camp for biomarker and anthropometric data collection. The questionnaires developed and used in the study are listed as supplemental material S1. Samples were collected first for HemoCue followed by DBS. A diagnosis of anemia was made based on $\mathrm{Hb}$ concentration cut-offs of $<11.0 \mathrm{~g} / \mathrm{dL}$ for children $6-59$ months and $<12.0 \mathrm{~g} / \mathrm{dL}$ for females $12-40$ years $^{17}$.

$\mathrm{Hb}$ measures were collected for the entire sample using HemoCue Hb 301 System using standard protocols developed for use in field settings ${ }^{11}$. Ten microliters of capillary blood were collected from each participant using disposable microcuvettes. For the subset of participants for whom DBS samples were also available, an additional five drops of capillary blood from the same puncture site were collected.. Capillary blood was collected by finger prick, and for children 6-12 months, by heel prick.

DBS samples were collected on Whatman 903 filter paper, which were then dried, stored in sealed plastic bags with desiccant, and transported to the National AIDS Research Institute (NARI) in Pune, located in the state of Maharashtra. There, DBS samples were stored at $-20^{\circ}$ centigrade and analyzed using the colorimetric indirect CMG method after elution in Drabkin's solution. A punch of $3.2 \mathrm{~mm}$ in diameter was cut from a single spot and eluted in Drabkin's solution for two hours on a shaker. Hb concentration was estimated in the eluent by measuring optical density on a spectrophotometer (Tecan Trading AG, Switzerland). Hb standards were included with every batch of samples and the standard curve with $\mathrm{R}^{2}$ value of $>0.95$ was considered acceptable. Low and normal level controls prepared from whole blood were also included in each batch of samples for monitoring internal quality control of the test. Method validation conducted prior to the study did not demonstrate volcano effect.

Enumeration teams underwent extensive training prior to the start of data collection. Practice exercises were conducted as per recommended training protocols ${ }^{11}$. Following practice, standardization exercises were conducted to help identify systematic errors in implementation of protocols for blood sample collection and with HemoCue machines. Interobserver and intra-observer correlation measures $<0.7$ were flagged as problematic, and further training and practice was advised for data collectors accordingly.

Sample selection for analysis. We included cases with both HemoCue and complete DBS samples available for children $(n=1695)$ and non-pregnant females $(n=1918)$, and excluded cases with implausible anthropometric data, as described in Fig. 1. DBS samples were considered unsuitable for analysis if the sample exhibited a serum ring, or if there was an insufficient quantity of blood ( $<75 \%$ of the spot was filled).

Statistical analysis. We first generated descriptive statistics of our sample to characterize sociodemographic characteristics as well as nutritional status indicated by height-for-age (HAZ) and weight-for-height $\mathrm{z}$-score $(\mathrm{WHZ})^{18}$ among children, body mass index-for-age $\mathrm{z}$-score (BFA) ${ }^{19}$ among adolescent girls $12-19$ years of age, and body mass index (BMI) among adult women 20-40 years of age. To test for systematic bias due to differences among 21 HemoCue machines used for the study, we conducted analysis of variance followed by Tukey and Scheffe pairwise comparisons.

We conducted paired t-tests of the difference of the two measures; a significant, non-zero difference between the means implies systematic bias. To explore the extent and patterns of agreement, we created Bland-Altman diagrams ${ }^{20}$, with $95 \%$ statistical limits of agreement (LoA), represented by \pm 1.96 standard deviations (SD) of the mean. For normally distributed difference values, it is expected that $95 \%$ of difference values would fall within these limits. It is important to note that the clinical LoA can be different from these and should be established prior to the analysis of a study ${ }^{21}$. We conducted a Pearson's correlation of the difference and mean of the HemoCue and DBS measures for statistical assessment of any patterns observed in the Bland-Altman plots; a significant correlation implies a dependence between the bias (difference of measures) and magnitude (mean of measures $)^{22}$. We assessed concordance between HemoCue and DBS measures by calculating Lin's concordance correlation coefficient (CCC) $)^{23}$. Lin's CCC $\left(\rho_{c}\right)$ is the appropriate test for agreement between two measures ${ }^{24}$ as it specifically considers how well the data fit around the 45-degree line of equality. CCC values ranging from 0.7 to 1.0 are considered good agreement, and values below 0.4 are considered poor agreement ${ }^{25}$.

For assessment of the binary outcome of interest (anemic/non-anemic), we tested whether the proportion of the samples who are classified as anemic differ significantly by method of measurement by conducting McNemar's 


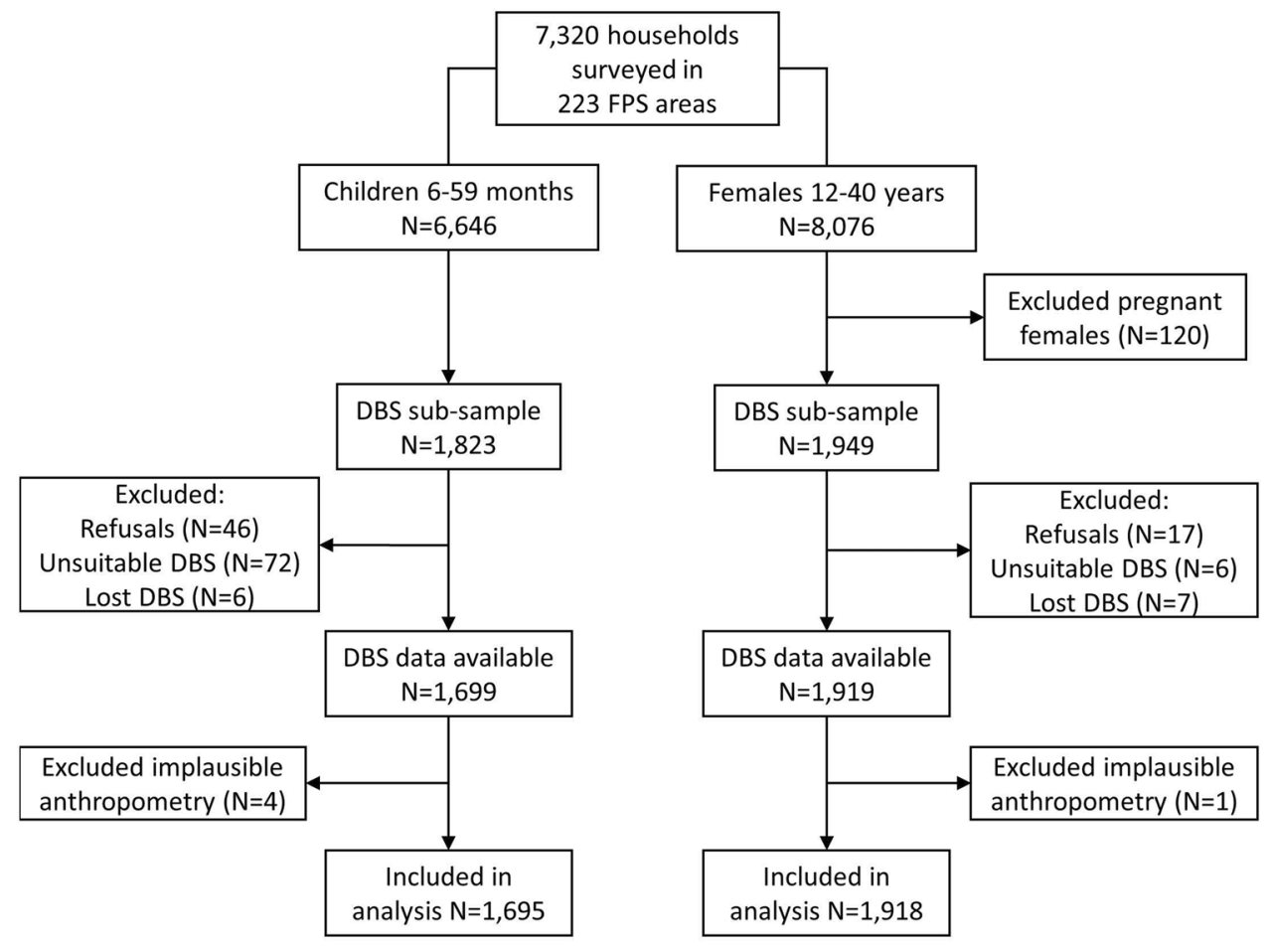

Figure 1. Sample selection flowchart for analysis of HemoCue and DBS Hb measurement agreement.

test of marginal homogeneity, a modification of the ordinary chi square test. Analyses were conducted separately for children 6-59 months $(n=1695)$, adolescent non-pregnant females $12-19$ years of age $(n=93)$, and adult non-pregnant females $20-40$ years of age $(n=1825)$. Since there were no differences observed between adolescent and adult females for our primary outcomes of $\mathrm{Hb}$ concentration and anemia prevalence (data not shown), we present these as combined results for all non-pregnant females 12-40 years of age. We conducted analyses in SAS 9.4, accounting for the multi-stage clustering survey design for descriptive statistics. Statistical significance was set at $\alpha=0.05$.

Ethics approval and consent to participate. Ethical review was conducted by Institutional Review Boards in India (IFMR) and the USA (Stanford University); the study was approved by the Indian Council of Medical Research (ICMR). Written informed consent was obtained from research participants. For children, written informed consent was obtained from caregivers. Fingerprints were obtained from research participants and caregivers who could not write their signature.

\section{Results}

Children who did not participate due to either refusal or unsuitable DBS samples were on average younger (9 months and 15 months, respectively) than children who successfully participated. Children for whom unsuitable DBS samples were collected had $0.5 \mathrm{~g} / \mathrm{dL}$ lower $\mathrm{Hb}$ values as measured by HemoCue, on average, compared to those children for whom valid DBS samples were collected for DBS analysis. There were no differences in mean age or $\mathrm{Hb}$ values as measured by HemoCue among females 12-40 years between successful participation and refusals or unsuitable DBS samples.

Study population characteristics. Over $99 \%$ of sampled females $12-40$ years belonged to a marginalized community (Scheduled Caste/Scheduled Tribe or Backward Class), and close to three-quarters completed secondary schooling or higher (Table 1). Among adolescent girls, $16.1 \%$ were thin and $17.2 \%$ were classified as overweight/obese. Among adult non-pregnant women, $14.4 \%$ were underweight and $36.8 \%$ were overweight or obese.

Our sample had slightly more male children than female children (Table 2). The prevalence of stunting was $22.8 \%$, and wasting was $13.6 \%$.

Measurement agreement. We did not observe systematic differences among HemoCue machines. Among children, mean $( \pm \mathrm{SD}) \mathrm{Hb}$ concentration was $10.79 \pm 1.34 \mathrm{~g} / \mathrm{dL}$ and $10.72 \pm 1.70 \mathrm{~g} / \mathrm{dL}$ by HemoCue and DBS, respectively. Among females 12-40 years, mean $\mathrm{Hb}( \pm \mathrm{SD})$ concentration was $11.64 \pm 1.42 \mathrm{~g} / \mathrm{dL}$ and $11.93 \pm 1.71 \mathrm{~g} / \mathrm{dL}$ by HemoCue and DBS, respectively. Paired t-tests between the two measures indicate the mean differences were significantly different from zero for both age groups (Table 3), implying bias. Concordance 


\begin{tabular}{|c|c|c|}
\hline & $\mathbf{n}$ & $\%$ \\
\hline \multicolumn{3}{|l|}{ Taluk } \\
\hline Bhuvanagiri & 745 & 38.8 \\
\hline Chidambaram & 1173 & 61.2 \\
\hline \multicolumn{3}{|l|}{ Age category } \\
\hline$<15$ years & 36 & 1.9 \\
\hline $15-19$ years & 57 & 3.0 \\
\hline $20-24$ years & 329 & 17.2 \\
\hline $25-29$ years & 790 & 41.2 \\
\hline 30-34 years & 517 & 26.9 \\
\hline$\geq 35$ years & 189 & 9.9 \\
\hline \multicolumn{3}{|l|}{ Education level } \\
\hline None & 48 & 2.5 \\
\hline Primary & 466 & 24.3 \\
\hline Secondary & 635 & 33.1 \\
\hline Higher & 769 & 40.1 \\
\hline \multicolumn{3}{|l|}{ Religion } \\
\hline Hindu & 1855 & 96.7 \\
\hline Muslim & 28 & 1.5 \\
\hline Christian & 35 & 1.8 \\
\hline \multicolumn{3}{|l|}{ Caste } \\
\hline General & 3 & 0.2 \\
\hline $\mathrm{SC} / \mathrm{ST}^{\mathrm{a}}$ & 874 & 45.7 \\
\hline Backward classes & 1037 & 54.2 \\
\hline \multicolumn{3}{|l|}{ BFA (12-19 years) ${ }^{b}$} \\
\hline Thin & 15 & 16.1 \\
\hline Normal & 62 & 66.7 \\
\hline Overweight/obese & 16 & 17.2 \\
\hline \multicolumn{3}{|c|}{ BMI category (20-40 years) ${ }^{c}$} \\
\hline Underweight & 262 & 14.4 \\
\hline Normal & 890 & 48.8 \\
\hline Overweight & 493 & 27,0 \\
\hline Obese & 178 & 9.8 \\
\hline Hb concentration, $\mathrm{g} / \mathrm{dL}$ & Mean & SD \\
\hline HemoCue & 11.64 & 1.42 \\
\hline DBS & 11.93 & 1.71 \\
\hline
\end{tabular}

Table 1. Sociodemographic characteristics and nutrition status of females $12-40$ years $(\mathrm{n}=1918) .{ }^{\mathrm{a}} \mathrm{SC} / \mathrm{ST}$ scheduled caste/scheduled tribe. ${ }^{b}$ Body mass index-for-age $\mathrm{z}$-score (BFA): $\mathrm{n}=93$, thin: $<-2$ BFA; normal: -2 to $\leq 1 \mathrm{BFA}$; overweight/obese: $>1$ BFA. ${ }^{\mathrm{C}}$ Body mass index (BMI): $\mathrm{n}=1823$, underweight: $<18.5 \mathrm{~kg} / \mathrm{m}^{2}$; normal: 18.5 to $<25 \mathrm{~kg} / \mathrm{m}^{2}$; overweight: 25 to $<30 \mathrm{~kg} / \mathrm{m}^{2}$; Obese: $\geq 30 \mathrm{~kg} / \mathrm{m}^{2}$.

between HemoCue and DBS was 0.67 for both groups, implying moderate agreement according to the cut-offs we used; alternate cut-offs have been proposed, further described in the discussion section.

Results from the analysis of the Bland-Altman plots (Fig. 2a,b) demonstrate that $95.1 \%$ of the difference values lay within the statistical LoA for both age groups as expected for normally distributed data. For children, the LoA were from -2.4 to $2.6 \mathrm{~g} / \mathrm{dL} ; 23$ of 1695 or $1.4 \%$ of values were above the upper limit and 60 or $3.5 \%$ of the values were below the lower limit. For females $12-40$ years, the LoA were from - 2.8 to $2.2 \mathrm{~g} / \mathrm{dL} ; 32$ of 1918 or $1.7 \%$ of values were above the upper limit and 62 or $3.2 \%$ of values were below the lower limit. Examination of the plots also shows that for both groups, as mean $\mathrm{Hb}$ concentration increases, the difference between HemoCue and DBS measures shifts from positive to negative values. The difference between HemoCue and DBS has a weak, negative correlation with the mean of both measures for children $(\mathrm{r}=-0.28, \mathrm{p}<0.0001)$ and females $12-40$ years $(r=-0.25, p<0.0001)$. That is, the difference between the measures depends on the magnitude of the measure.

To explore this further, we present the average difference between HemoCue and DBS by Hb concentration interval, shown in Table 4. Among children, mean difference values at the lower $\mathrm{Hb}$ concentration intervals tend to be positive and decrease to negative values at higher $\mathrm{Hb}$ concentration intervals; this pattern is not as apparent among females 12-40 years. For both groups, variability in the differences increases with increasing magnitude.

Anemia classification. Among children, 50\% and 56\% classified as anemic according to HemoCue and DBS, respectively (Table 5). Among females $12-40$ years, 55\% classified as anemic according to HemoCue and 


\begin{tabular}{|c|c|c|}
\hline & n & $\%$ \\
\hline \multicolumn{3}{|l|}{ Age category } \\
\hline 6-11 months & 117 & 6.9 \\
\hline $12-17$ months & 189 & 11.2 \\
\hline $18-23$ months & 169 & 10.0 \\
\hline $24-29$ months & 185 & 10.9 \\
\hline 30-35 months & 178 & 10.5 \\
\hline 36-41 months & 208 & 12.3 \\
\hline $42-47$ months & 178 & 10.5 \\
\hline $48-53$ months & 233 & 13.7 \\
\hline 54-59 months & 238 & 14.0 \\
\hline \multicolumn{3}{|l|}{ Sex } \\
\hline Male & 917 & 54.1 \\
\hline Female & 777 & 45.8 \\
\hline \multicolumn{3}{|l|}{ Stunting $^{\mathrm{a}}$} \\
\hline Severely stunted & 78 & 4.6 \\
\hline Moderately stunted & 306 & 18.2 \\
\hline Not stunted & 1297 & 77.2 \\
\hline \multicolumn{3}{|l|}{ Wasting $^{\mathrm{b}}$} \\
\hline Severely wasted & 22 & 1.3 \\
\hline Moderately wasted & 206 & 12.3 \\
\hline Not wasted & 1449 & 86.3 \\
\hline Hb concentration, $g / d L$ & Mean & SD \\
\hline HemoCue & 10.79 & 1.34 \\
\hline DBS & 10.72 & 1.70 \\
\hline
\end{tabular}

Table 2. Age, sex, and nutrition status of children 6-59 months $(\mathrm{n}=1695)$. ${ }^{\mathrm{a}}$ Severely stunted: $<-3 \mathrm{HAZ}$;

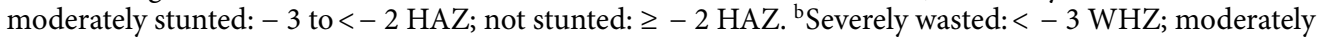
wasted: -3 to $<-2$ WHZ; not wasted: $\geq-2$ WHZ.

\begin{tabular}{|l|l|l|l|l|l|l|l|}
\hline & \multicolumn{2}{|l|}{ Mean difference $\mathbf{( g / d L )}$} & Range & \multicolumn{2}{l|}{ Paired t-test } & \multicolumn{2}{l|}{ Concordance } \\
\cline { 2 - 8 } & HemoCue-DBS & $\mathbf{( 9 5 \%} \mathbf{C I})$ & $(\mathbf{m i n}, \mathbf{m a x})$ & $\mathbf{t}$ & $\mathbf{p}$-value & $\boldsymbol{\rho}_{\mathbf{c}}$ & $\mathbf{( 9 5 \%} \mathbf{C I})$ \\
\hline Children 6-59 months & 0.06 & $(0.002,0.12)$ & $(-7.49,4.51)$ & 2.02 & 0.043 & 0.67 & $(0.65,0.71)$ \\
\hline Females 12-40 years & -0.29 & $(-0.34,-0.23)$ & $(-6.48,6.59)$ & -9.91 & $<0.0001$ & 0.67 & $(0.64,0.69)$ \\
\hline
\end{tabular}

Table 3. Measures of agreement between HemoCue and DBS, children 6-59 months and females 12-40 years. CI confidence interval.

$47 \%$ classified as anemic according to DBS. For both groups, the null hypothesis of marginal homogeneity was rejected (McNemar's $\chi^{2}: 42.5, p<0.0001$ and McNemar's $\chi^{2}: 18.5, p<0.0001$, respectively), indicating that the proportion of positive cases from the two methods are significantly different, more so in females $12-40$ years than in children. In Fig. 3 we show overlaid histograms of HemoCue and DBS Hb concentration, as well as the anemia threshold, to illustrate the different patterns in children 6-59 months and females 12-40 years. In the latter group, the HemoCue values distribution is shifted to the left of the anemia threshold compared to the shift to the right of the threshold for DBS values. In children, there is greater overlap in the distributions of HemoCue and DBS Hb values.

\section{Discussion}

Around $50 \%$ of children 6-59 months and 55\% of non-pregnant females $12-40$ years in our study sample were diagnosed as anemic using the point-of-care device HemoCue; these prevalence estimates are consistent with national ${ }^{3}$ and regional ${ }^{26}$ data. Our results demonstrate that there is moderate statistical agreement between indirect CMG using DBS and HemoCue in capillary $\mathrm{Hb}$ assessment among both children 6-59 months and females 12-40 years of age. Concordance between the two measures was 0.67 for both groups, indicating moderate agreement according to criteria used in previous research ${ }^{25}$; however, McBride has proposed alternative cut-offs $^{27}$, for which all CCC values below 0.90 are considered poor. Lin's CCC is the appropriate method of assessing agreement as it provides the extent of fit around the line of perfect agreement, whereas Pearson's correlation coefficient provides the extent of fit around any line ${ }^{24}$.

The bias observed between the two measures seems to operate inversely for children and females 12-40 years. HemoCue measures $0.06 \mathrm{~g} / \mathrm{dL}$ higher than DBS, on average, for children and are $0.29 \mathrm{~g} / \mathrm{dL}$ lower than DBS for 


\section{a. Children 6-59 months}

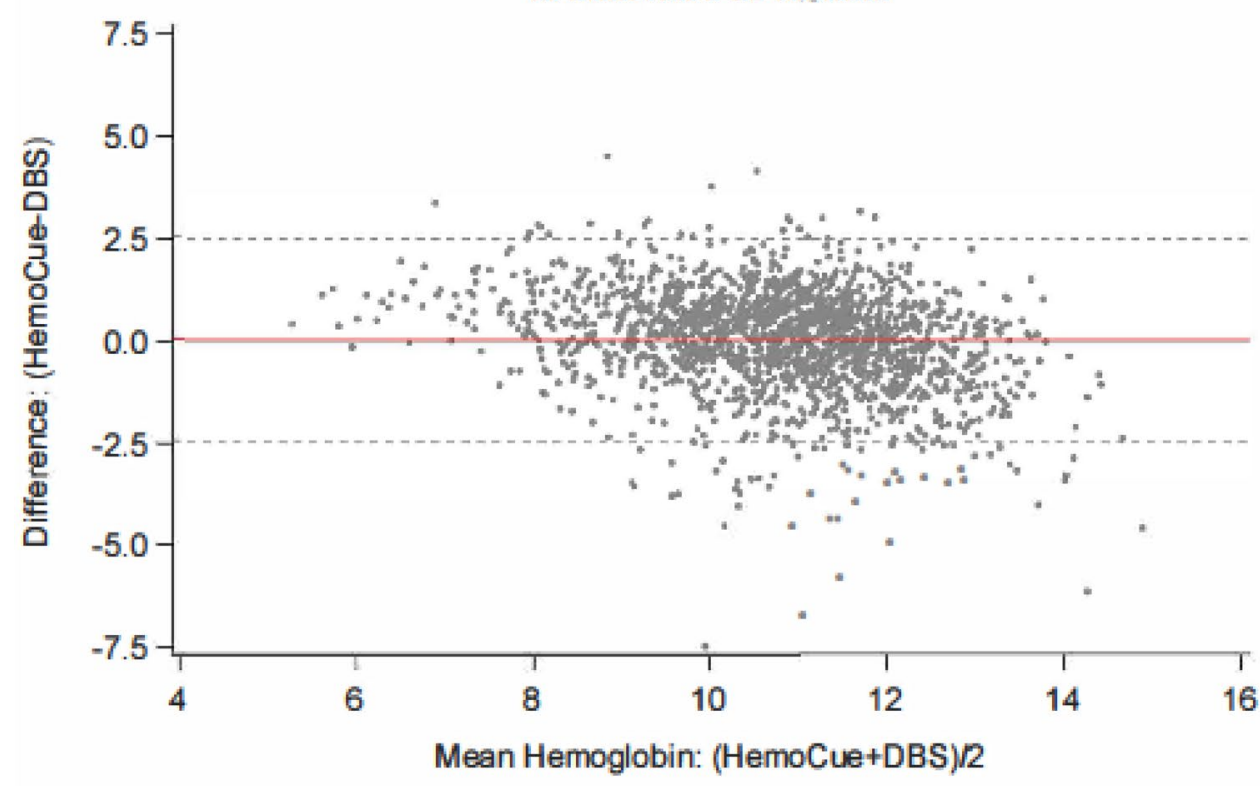

b. Females $12-40$ years

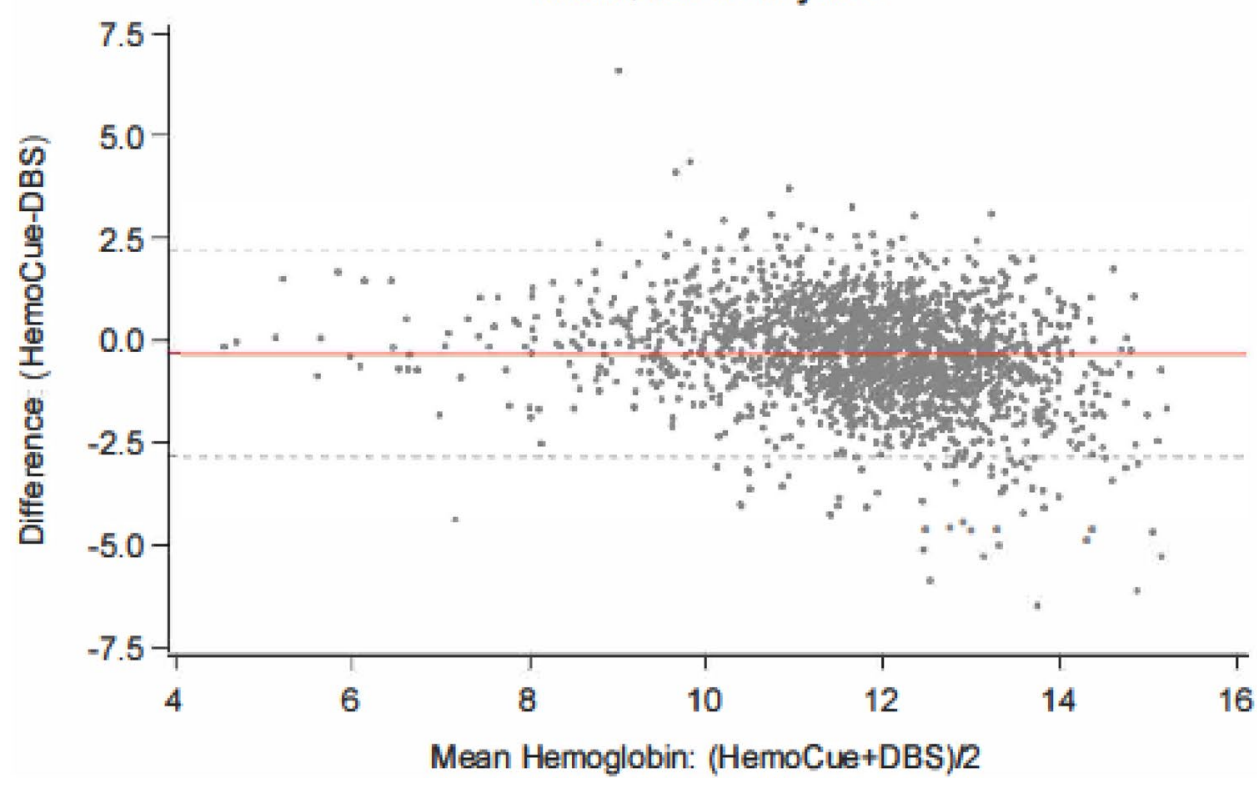

Figure 2. Bland-Altman plot of HemoCue and DBS Hb measures, children 6-59 months (a) and females 12-40 years (b). The red line represents the mean difference; the dashed grey lines represent $\pm 1.96 \mathrm{SD}$ of the mean difference indicating the statistical limits.

\begin{tabular}{|l|l|l|l|l|}
\hline \multirow{2}{*}{ Hb interval $^{\mathbf{a}}$} & \multicolumn{3}{|l|}{ Children 6-59 } & \multicolumn{2}{l|}{ Females 12-40 years } \\
\cline { 2 - 5 } & $\mathbf{n}$ & Mean difference ${ }^{\mathbf{b}} \pm$ SD & $\mathbf{n}$ & Mean difference $^{\mathbf{b}} \pm$ SD \\
\hline$<8 \mathrm{~g} / \mathrm{dL}$ & 66 & $0.98 \pm 0.80$ & 33 & $-0.06 \pm 1.14$ \\
\hline 8 to $<10 \mathrm{~g} / \mathrm{dL}$ & 402 & $0.35 \pm 1.18$ & 160 & $0.29 \pm 1.19$ \\
\hline 10 to $<12 \mathrm{~g} / \mathrm{dL}$ & 912 & $0.08 \pm 1.21$ & 805 & $-0.09 \pm 1.17$ \\
\hline$\geq 12 \mathrm{~g} / \mathrm{dL}$ & 315 & $-0.55 \pm 1.28$ & 920 & $-0.57 \pm 1.29$ \\
\hline
\end{tabular}

Table 4. Mean differences by Hb concentration interval, children 6-59 months and females 12-40 years. ${ }^{a}$ Average of DBS and HemoCue measures. ${ }^{b}$ HemoCue-DBS. 


\begin{tabular}{|l|l|l|l|l|l|l|}
\hline & \multicolumn{4}{|l|}{ Children 6-59 months } & \multicolumn{2}{l|}{ Females 12-40 years } \\
\cline { 2 - 7 } & Anemia DBS, $\mathbf{n}$ & No Anemia DBS, $\mathbf{n}$ & Total, $\mathbf{n}$ (\%) & Anemia DBS, n & No Anemia DBS, $\mathbf{n}$ & Total, $\mathbf{n}(\%)$ \\
\hline Anemia HemoCue, $\mathbf{n}$ & 699 & 183 & $852(50.3)$ & 703 & 360 & $1063(55.4)$ \\
\hline $\begin{array}{l}\text { No anemia HemoCue, } \\
\text { n }\end{array}$ & 275 & 568 & $843(49.7)$ & 205 & 650 & $855(44.6)$ \\
\hline Total n, (\%) & $944(55.7)$ & $751(44.3)$ & $1695(100.0)$ & $908(47.3)$ & $1010(52.7)$ & $1918(100.0)$ \\
\hline
\end{tabular}

Table 5. Anemia classification ${ }^{\mathrm{a}}$ by HemoCue and DBS, children 6-59 months and females 12-40 years. ${ }^{\mathrm{a}}$ Anemia is classified as $\mathrm{Hb}$ concentration $<11.0 \mathrm{~g} / \mathrm{dL}$ for children and $<12.0 \mathrm{~g} / \mathrm{dL}$ for females.

\section{a. Children 6-59 months}

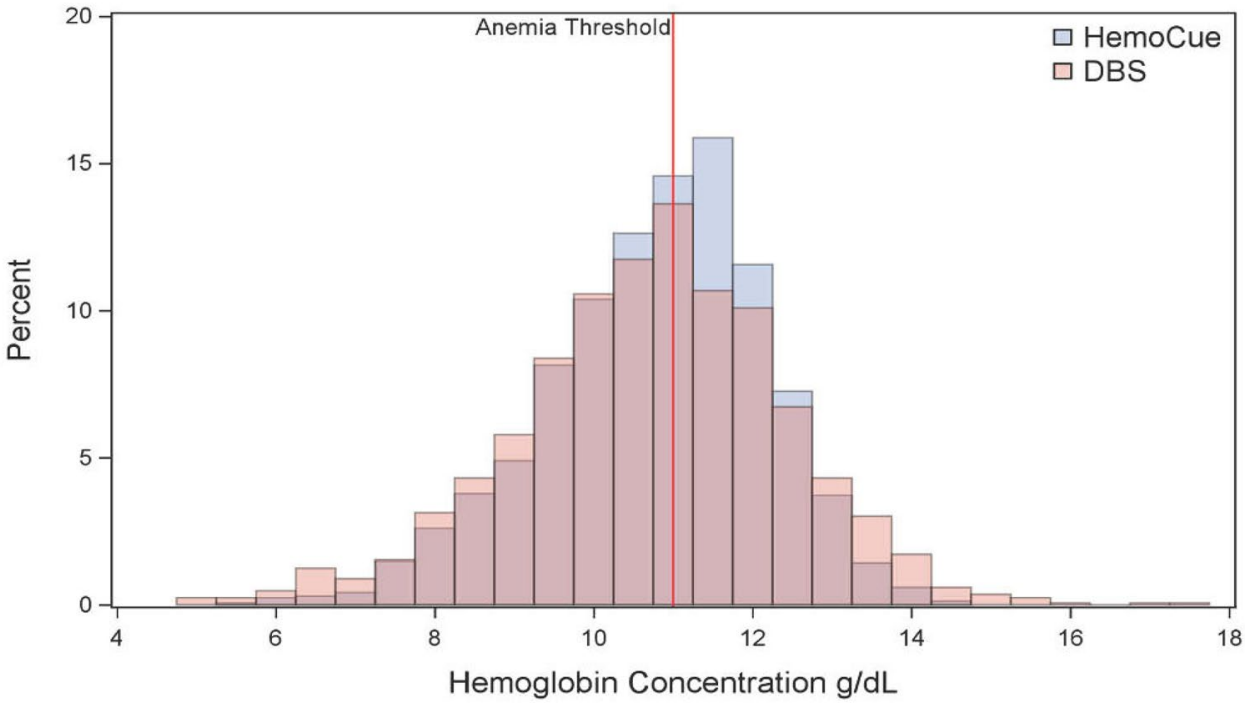

b. Females $12-40$ years

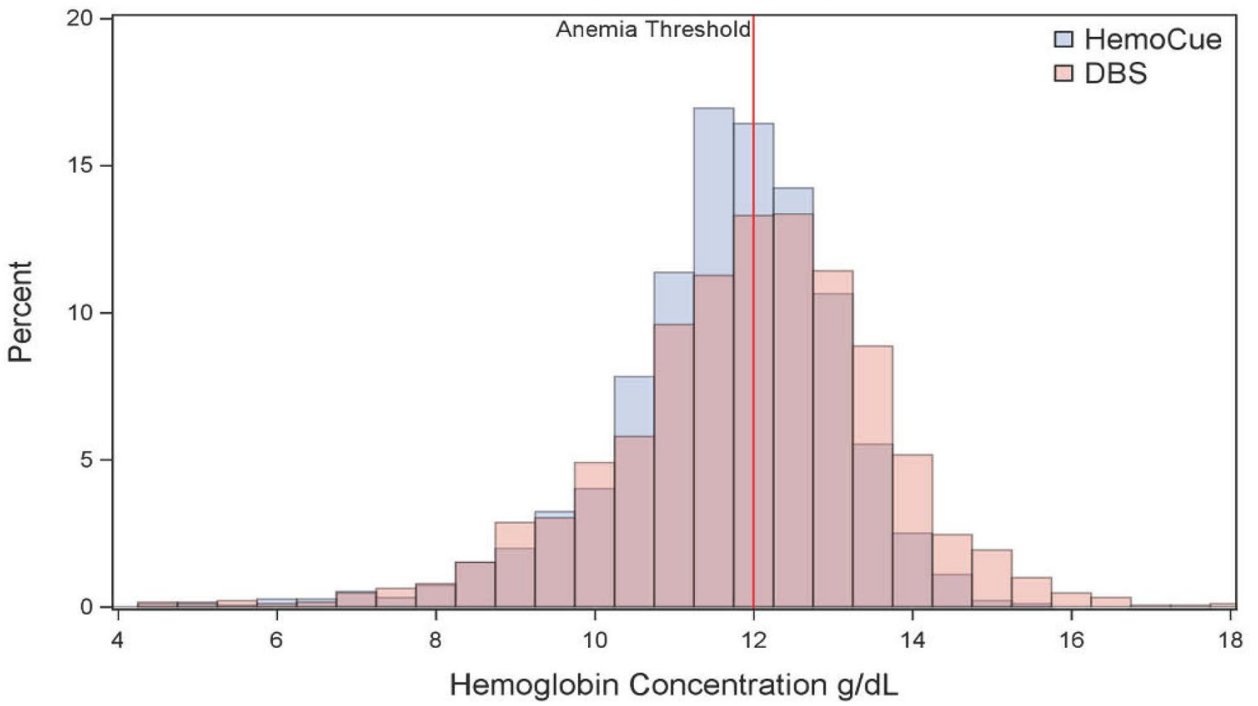

Figure 3. Overlaid histograms for $\mathrm{Hb}(\mathrm{g} / \mathrm{dL}$ ) assessed by HemoCue and DBS for (a) children 6-59 months and (b) females 12-49 years, with the threshold for anemia shown in red.

females, on average. While these differences were significant, it is important to note that our study had a large sample size and was therefore powered to detect small differences. Indeed, the magnitude of difference is minor and arguably clinically insignificant, particularly among children. At the same time, the observed bias translates to significantly different anemia prevalence estimates at the population level given the distribution of $\mathrm{Hb}$ values around the age-specific anemia thresholds. Measures by HemoCue result in a lower anemia prevalence compared to DBS by over five percentage points for children, and $29 \%$ of children who are classified as anemic according 
to DBS would classify as normal using HemoCue. Conversely, measures by HemoCue result in a higher anemia prevalence compared to DBS by eight percentage points for females $12-40$ years; around 34\% who are classified as anemic according to HemoCue would classify as normal using DBS.

Overall, the differences by method we observed in both mean $\mathrm{Hb}$ measurements and anemia prevalence are lower than those reported by previous studies ${ }^{13-15}$; in contrast to our findings, these studies also observed lower $\mathrm{Hb}$ concentration by indirect CMG using DBS compared to HemoCue for capillary blood samples among adult women. Potential explanations include substantial variability of $\mathrm{Hb}$ in capillary blood depending on site of collection and over time for example ${ }^{28}$, differing climatic and environmental conditions, or varying specimen collection and quality control protocols ${ }^{11}$. Ours is the first study to compare these two methods among children.

The 95\% LoA represent the limits within which $95 \%$ of normally distributed difference values are expected to lie; in our study, they range from -2.4 to $2.6 \mathrm{~g} / \mathrm{dL}$ and -2.8 to $2.2 \mathrm{~g} / \mathrm{dL}$ for children and females $12-40$ years, respectively. In other words, for $95 \%$ children for example, one method of measurement would range from 2.4 less to $2.6 \mathrm{~g} / \mathrm{dL}$ more than the other method of measurement ${ }^{29}$. These are statistically derived, however, and may be unacceptable for clinical method agreement. The threshold criteria for hemoglobin estimation evaluation established by the Clinical Laboratory Improvement Amendments ${ }^{30}$ is $\pm 7 \%$ of the reference method. We were unable to assess the percent difference with a reference method, mentioned in the limitations below; however, the mean measurements of the two methods we compared were within $\pm 7 \%$ of each other for both age groups. We also found the difference between HemoCue and DBS measures varies with Hb concentration magnitude, especially among children. Within this group, HemoCue measures appear higher than DBS at lower Hb concentration levels, and lower than DBS at higher $\mathrm{Hb}$ concentration levels, reinforcing our finding that HemoCue results in a lower anemia prevalence compared to DBS among children. Varying HemoCue performance by magnitude among children has been previously observed ${ }^{16}$. This might be partially explained by varying quality of blood samples. Blood collection among children is often challenging. In our study, over $90 \%$ of DBS samples that were unsuitable for analysis were among children. 'Milking', or application of pressure on the site of the puncture to stimulate blood flow should not be done since it results in hemodilution by increasing plasma and interstitial fluid in the sample, thereby lowering $\mathrm{Hb}$ concentration readings ${ }^{28}$. Although this process was discouraged during trainings, if 'milking' did inadvertently occur, we would expect $\mathrm{Hb}$ measures by DBS to be lower as we observed, since DBS collection occurred after HemoCue for all research participants. Varying the order of sample collection by method would have allowed testing of the effects of this potential source of measurement error; we recommend this to be done in future studies. However, we would have expected this trend across the $\mathrm{Hb}$ magnitude spectrum, not only at the lower end. Our study highlights the need for research on methodological validation over the $\mathrm{Hb}$ concentration range especially among children.

The choice of method of anemia assessment can have a substantial effect at the individual level with respect to appropriate anemia classification, referral and treatment, as well as at regional levels with respect to prevalence estimates and resource allocation. In India, national programs ${ }^{31}$ provide prophylactic iron and folic acid supplementation to children, adolescent boys and girls, women of reproductive age, and pregnant and lactating women. The country's anemia reduction strategy also calls for periodic screening and testing for anemia in all age groups, and appropriate referral and treatment based on mild, moderate and severe anemia classification. Currently, anemia is screened at the community level using clinical pallor assessment ${ }^{32}$ and tested at the primary health clinic and hospital levels utilizing Sahli's method, for which capillary blood is collected by pipette and mixed with hydrochloric acid and distilled water until the formed brown acid hematin matches the color of the standard. It is inexpensive and simple, but is subjective as the method is based on color comparison ${ }^{33}$. Studies assessing accuracy of Sahli's method have found high sensitivity (84-92\%) but low specificity (39-63\%) compared to HemoCue ${ }^{34,35}$. Strategy documentation suggests that digital hemoglobinometers will be adopted for $\mathrm{Hb}$ measurement ${ }^{31}$. The eventual shift in measurement method will have important implications in the context of drawing conclusions across sources of monitoring data, national level statistics, and micro-level studies that utilize different and inconsistent methods of $\mathrm{Hb}$ measurement.

Our study limitations include the absence of a gold standard reference measure thus limiting our ability to assess and compare the accuracy of HemoCue and DBS. There are very few community-based studies validating capillary samples analyzed by the HemoCue 301 model. Existing research has found that compared the gold standard, HemoCue overestimates Hb levels by $0.16 \mathrm{~g} / \mathrm{dL}$ among adults ${ }^{36}$ and by $0.87 \mathrm{~g} / \mathrm{dL}$ among children $^{37}$, resulting in lower anemia prevalence. The accuracy of $\mathrm{Hb}$ measurement using indirect CMG of capillary blood was assessed in only one field-based study among adult women which found that indirect CMG results in a $0.67 \mathrm{~g} /$ $\mathrm{dL}$ lower $\mathrm{Hb}$ level compared to direct $\mathrm{CMG}^{13}$, leading to a higher anemia prevalence. Based on the available data, we were unable to assess variability by surveyor, which may have contributed to variability in HemoCue and DBS measures. Additionally, we were unable to report reliability for either method in our study. Previous research has reported intra-sample variability higher for DBS (SD of $1.0 \mathrm{~g} / \mathrm{dL}$ ) than for direct CMG (SD of $0.4 \mathrm{~g} / \mathrm{dL})^{13}$. Evidence on reliability of capillary HemoCue has found that while differences between repeat samples are nonsignificant, coefficients of variation range from 3.9 to $7.0 \%{ }^{16,28}$. Comparing the reliability of two field-friendly methods, DBS and HemoCue, would be a valuable future analysis that will contribute to the interpretation of changes over time within individuals as well as population level trends.

To the best of our knowledge, this is the first study to assess agreement between HemoCue and indirect CMG using DBS among children and furthermore, compare agreement with an adolescent and adult female population. While capillary HemoCue is a widely used field-friendly method, machines are relatively costly and validity and reliability remain questionable. DBS presents an alternative method to HemoCue for use in field settings, one which can allow for testing of multiple analytes with a single sample, for example measures of iron status and inflammation which would be immensely valuable in contexts such as India. However, further research is required to assess factors that influence DBS accuracy and reliability for Hb estimation, especially among children, an important target group for anemia prevention and control. 


\section{Conclusions}

Our study found moderate agreement between two methods that are feasible in field settings, HemoCue and indirect CMG using DBS. The bias we observed was small but operated differently for females 12-40 years and children 6-59 months, and is dependent on $\mathrm{Hb}$ magnitude, which should be further explored. In addition, the two methods resulted in differing anemia prevalence estimates, demonstrating that the choice of Hb assessment method has important implications for individual anemia diagnosis and population prevalence estimates. Accurate and field-friendly $\mathrm{Hb}$ assessment methods are necessary to meaningfully identify targets and track progress towards anemia reduction. Indirect DBS is a potential alternative to the widely used HemoCue device. However, additional studies with comparison to the reference method are needed. We recommend further research to understand utilization of DBS methods for $\mathrm{Hb}$ assessment, especially among young children.

\section{Data availability}

The data set used in this article is available from the corresponding author and other Principal Investigators upon reasonable request.

Received: 23 July 2020; Accepted: 8 April 2021

Published online: 29 April 2021

\section{References}

1. Balarajan, Y., Ramakrishnan, U., Ozaltin, E., Shankar, A. H. \& Subramanian, S. V. Anaemia in low-income and middle-income countries. Lancet 378, 2123-2135 (2011).

2. Ministry of Health and Family Welfare (MoHFW) Government of India. Comprehensive National Nutrition Survey (CNNS) national report. https://www.nutritionindia.info/ (2019).

3. International Institute of Population Sciences. National Family Health Survey (NFHS-4), 2015-16. http://rchiips.org/nfhs/NFHS4Report.shtml (2017).

4. Anand, T., Rahi, M., Sharma, P. \& Ingle, G. K. Issues in prevention of iron deficiency anemia in India. Nutrition 30, 764-770 (2014).

5. Nguyen, P. H., Scott, S., Avula, R., Tran, L. M. \& Menon, P. Trends and drivers of change in the prevalence of anaemia among 1 million women and children in India, 2006 to 2016. BMJ Glob. Health. 3, e001010 (2018).

6. Zwart, A., et al. Recommendations for reference method for haemoglobinometry in human blood (ICSH standard 1995) and specifications for international haemiglobinocyanide standard (4th edition). J. Clin. Pathol. 49, 271-274 (1996).

7. Neufeld, L. M. et al. Hemoglobin concentration and anemia diagnosis in venous and capillary blood: Biological basis and policy implications. Ann. NY Acad. Sci. 1450, 172-189 (2019).

8. Sanchis-Gomar, F., Cortell-Ballester, J., Pareja-Galeano, H., Banfi, G. \& Lippi, G. Hemoglobin point-of-care testing: The HemoCue system. J. Lab. Autom. 18, 198-205 (2013).

9. Gwetu, T., Chhagan, M., Craib, M., \& Kauchali, S. Hemocue validation for the diagnosis of anaemia in children: A semi-systematic review. Pediat. Therapeut. 4, (2013).

10. Whitehead, R. D. Jr. et al. Effects of preanalytical factors on hemoglobin measurement: A comparison of two HemoCue(R) pointof-care analyzers. Clin. Biochem. 50, 513-520 (2017).

11. Burger, S. \& Pierre-Louis, J. A procedure to estimate the accuracy and reliability of HemoCue measurements of survey workers. http://citeseerx.ist.psu.edu/viewdoc/download?doi=10.1.1.607.2604\&rep=rep1\&type=pdf (2003).

12. McDade, T. W., Williams, S. \& Snodgrass, J. J. What a drop can do: Dried blood spots as a minimally invasive method for integrating biomarkers into population-based research. Demography 44, 899-925 (2007).

13. Sari, M. et al. Estimating the prevalence of anaemia: A comparison of three methods. Bull. World Health Organ. 79, 506-511 (2001).

14. Mohanram, M., Rao, G. V. R. \& Sastry, J. G. A comparative study on prevalence of anaemia in women by cyanmethaemoglobin and HemoCue methods. Indian J. Community Med. 27, 58-61 (2002).

15. Pathak, P., Kapoor, S., Dwivedi, S., Singh, P. \& Kapil, U. Comparison of hemoglobin estimates from filter paper cyanmethemoglobin and HemoCue methods. Indian J. Community Med. 29, 149 (2004).

16. Neufeld, L. et al. Hemoglobin measured by Hemocue and a reference method in venous and capillary blood: A validation study. Salud Publica Mex. 44, 219-227 (2002).

17. World Health Organization. Haemoglobin concentrations for the diagnosis of anaemia and assessment of severity. https://www. who.int/vmnis/indicators/haemoglobin/en/ (2011).

18. WHO Multicentre Growth Reference Study Group. WHO Child Growth Standards based on length/height, weight and age. Acta Paediatr. Suppl. 450, 76 (2006).

19. De Onis, M. et al. Development of a WHO growth reference for school-aged children and adolescents. Bull. World Health Organ. 85, 660-667 (2007).

20. Bland, J. M. \& Altman, D. Statistical methods for assessing agreement between two methods of clinical measurement. Lancet 327, 307-310 (1986).

21. Bland, J. M. \& Altman, D. G. Measuring agreement in method comparison studies. Stat. Methods Med. Res. 8, 135-160 (1999).

22. Altman, D. G. \& Bland, J. M. Measurement in medicine: The analysis of method comparison studies. J. R. Stat. Soc. Ser. D (The Statistician). 32, 307-317 (1983).

23. Lin, L. A concordance correlation coefficient to evaluate reproducibility. Biometrics 45, 255-268 (1989).

24. Watson, P. \& Petrie, A. Method agreement analysis: A review of correct methodology. Theriogenology 73, 1167-1179 (2010).

25. Quinn, C., Haber, M. J. \& Pan, Y. Use of the concordance correlation coefficient when examining agreement in dyadic research. Nurs. Res. 58, 368-373 (2009).

26. Little, M. et al. Burden and determinants of anemia in a rural population in south India: A cross-sectional study. Anemia. 2018, 7123976 (2018).

27. McBride, G. B. A proposal for strength-of-agreement criteria for Lin's concordance correlation coefficient. https://www.medcalc. org/download/pdf/McBride2005.pdf (2005).

28. Morris, S. S. et al. Precision, accuracy, and reliability of hemoglobin assessment with use of capillary blood. Am. J. Clin. Nutr. 69, 1243-1248 (1999).

29. Bunce, C. Correlation, agreement, and Bland-Altman analysis: Statistical analysis of method comparison studies. Am. J. Ophthalmol. 148, 4-6 (2009).

30. Department of Health and Human Services. Fed. Reg. 57, 7002-7186 (1992).

31. Ministry of Health and Family Welfare Government of India. Anemia Mukt Bharat Intensified National Iron Plus Initiative (I-NIPI): Operational guidelines for programme managers. https://anemiamuktbharat.info/resources/ (2018).

32. Kapil, U., Kapil, R. \& Gupta, A. Prevention and control of anemia amongst children and adolescents: Theory and practice in India. Indian J. Pediatr. 86, 523-531 (2019). 
33. Srivastava, T., Negandhi, H., Neogi, S. B., Sharma, J. \& Saxena, R. Methods for hemoglobin estimation: A review of “what works”. J. Hematol. Transfus. 2, 1028 (2014).

34. Barduagni, P., Ahmed, A. S., Curtale, F., Raafat, M. \& Soliman, L. Performance of Sahli and colour scale methods in diagnosing anaemia among school children in low prevalence areas. Trop. Med. Int. Health. 8, 615-618 (2003).

35. Patil, P. J., Thakare, G. V. \& Patil, S. P. Variability and accuracy of Sahli's method in estimation of haemoglobin concentration. Natl. J. Integr. Res. Med. 4, 38-44 (2013).

36. Jaggernath, M. et al. Diagnostic accuracy of the HemoCue Hb 301, STAT-Site MHgb and URIT-12 point-of-care hemoglobin meters in a central laboratory and a community based clinic in Durban South Africa. PLoS ONE 11, e0152184 (2016).

37. Hinnouho, G. M. et al. Comparison of haemoglobin assessments by HemoCue and two automated haematology analysers in young Laotian children. J. Clin. Pathol. 71, 532-538 (2018).

\section{Acknowledgements}

The baseline survey was carried out by The Abdul Latif Jameel Poverty Action Lab (J-PAL) of South Asia.

\section{Author contributions}

R.M. conceptualized the analysis. R.M. and R.P.R. made substantial contributions to the design of the study. A.S., R.B. and S.K. made substantial contributions to laboratory analysis and R.V.M. led training sessions for HemoCue blood collection. R.P.R. led the statistical analysis of data. A.M., G.M., A.T. and R.M. provided the data. All authors contributed to interpretation of data. R.P.R. and R.M. drafted the manuscript. All authors critically revised the manuscript.

\section{Funding}

The data were collected as part of the baseline for a study "Reducing Anemia through Food Fortification at Scale" and was supported by the Tamil Nadu government, the Tata Trusts, the Global Innovation Fund and King Philanthropies through grants to Grant Miller (Stanford University), Aprajit Mahajan (University of California Berkeley), Alessandro Tarozzi (Universitat Pompeu Fabra) and Reynaldo Martorell (Emory University). The funders had no role in the study.

\section{Competing interests}

The authors declare no competing interests.

\section{Additional information}

Supplementary Information The online version contains supplementary material available at https://doi.org/ 10.1038/s41598-021-88425-y.

Correspondence and requests for materials should be addressed to R.M.

Reprints and permissions information is available at www.nature.com/reprints.

Publisher's note Springer Nature remains neutral with regard to jurisdictional claims in published maps and institutional affiliations.

(c) Open Access This article is licensed under a Creative Commons Attribution 4.0 International cc) License, which permits use, sharing, adaptation, distribution and reproduction in any medium or format, as long as you give appropriate credit to the original author(s) and the source, provide a link to the Creative Commons licence, and indicate if changes were made. The images or other third party material in this article are included in the article's Creative Commons licence, unless indicated otherwise in a credit line to the material. If material is not included in the article's Creative Commons licence and your intended use is not permitted by statutory regulation or exceeds the permitted use, you will need to obtain permission directly from the copyright holder. To view a copy of this licence, visit http://creativecommons.org/licenses/by/4.0/.

(C) The Author(s) 2021 